\title{
Health Promotion Among Families Having a Newborn Baby
}

\author{
Shefaly Shorey
}

\begin{abstract}
Pregnancy, childbirth, and the postpartum period are the stressful transition periods to parenthood. With medicalization of perinatal period, parents feel left out and less confident in their parenthood journey, which may pose serious threats to the family dynamics. Salutogenesis theory offers the potential to influence a shift away from negative health outlooks and outcomes, medicalization of childbirth, toward health promotion and positive well-being focus for maternity care services design and delivery in the future.
\end{abstract}

\section{Keywords}

Salutogenesis $\cdot$ Health promotion $\cdot$ Perinatal period

\subsection{Introduction}

This chapter describes the need of health promotion strategies among families having a newborn baby. The focus of the utilization of the Salutogenesis theory in health promotion and future directions in health promotion research has

\footnotetext{
S. Shorey $(\bowtie)$

Alice Lee Centre for Nursing Studies, National University of Singapore, Singapore, Singapore e-mail: nurssh@nus.edu.sg
}

been discussed. The following discussions are based on the research on perinatal mental health, trends in childbirth, health promotion, and, particularly, the integration of the Salutogenesis theory in health promotion during the perinatal period. The extensive discussions constitute a basis for development of future policies or initiatives to promote health promotion among families having a newborn that meet the standards for health care education and research.

\subsection{Perinatal Mental Health}

The perinatal period spans from the start of one's pregnancy to the first 12 months after childbirth [1]. This period is a stressful and remarkably dynamic period of growth that poses significant challenges for both pregnant women and their partners [2]. Right from pregnancy till after child birth, the perinatal period is a complex phenomenon that consists of a myriad of adjustments to physical, social, and emotional lifestyles, that influence the overall well-being of the parents [3].

There is increasing research on parental perinatal mental health, in which systematic reviews revealed that $6.5 \%$ to $12.9 \%$ of pregnant and postpartum mothers experienced depression and anxiety [4, 5]. Likewise, depression and anxiety have been reported to be the most common mental health issues faced by fathers during the perinatal period $[6,7]$. Existing meta-analyses 
have reported that the prevalence rate for paternal depression during the perinatal period was approximately $8.4 \%$ [8] and the prevalence of anxiety in fathers ranged between $3.4 \%$ and $25 \%$ during the prenatal period, to $2.4 \%$ and $51 \%$ during the postnatal period [9]. The striking prevalence of mental health issues among both mothers and fathers urge the need for a focus shift toward familial health promotion throughout the perinatal period.

In recent years, the experiences and needs of both mothers and fathers during the perinatal period have been explored. During pregnancy, mothers reported that it was an emotional roller-coaster ride that made them feel overwhelmed with the evolving pregnancy needs [10]. During postpartum, mothers felt varied emotional issues and many of them felt neglected to seek any help [10]. Mothers also experienced unpreparedness, anxiety, stress over infant care, breast-feeding concerns, and physical discomfort [11]. Existing barriers such as stigma, shame, and the lack of time and interactions with health care professionals prevented mothers from disclosing their feelings and needs [10, 12]. Hence, mothers expressed their need for (1) continuity of care, (2) mental health enquiries to include less common disorders on top of depression and anxiety, (3) culturally appropriate postnatal practices in infant care and upbringing, and (4) professional support needs, i.e. more information with regard to health care services $[10,11,13]$. Similarly, fathers exhibited negative feelings and psychological difficulties during the perinatal period. They expressed feelings of insecurity and inadequacy, felt isolated and excluded from the events that happened throughout the perinatal period [14-18]. Fathers also expressed stress, anxiety, depression, and role strain and conflict while they tried to cope with other role demands such as their jobs, parental stress from their children (e.g., fear of developmental problems), and conflict with inlaws and partners [14, 19, 20]. Therefore, fathers reported their needs: (1) including more educational information about pregnancy and parenting (during antenatal classes), (2) engaging them with other experienced fathers through small groups and focused group discussions [14, 17,
18], (3) having topics of discussion beyond the infant and partner care such as to include mental health topics $[14,20]$, (4) ensuring continuity of care after hospital discharge after the childbirth to seek timely help from health care professionals, and (5) incorporating technology (e.g., mobile health applications) to receive information covering mental health [21]. These evidences highlight the need to explore health promotion strategies among parents with a newborn to ensure smooth transition to parenthood and overall well-being of new parents and eventually their entire families.

\subsection{Trends in Childbirth}

Before the twentieth century, childbirth was considered a natural, normal, female-centred event, and hospital birth was uncommon [22, 23]. Female midwives predominantly helped with the childbirth, whereas men were rarely involved, and if they did, it was mainly during difficult deliveries [22]. In the nineteenth and twentieth centuries, medical influence on childbirth exaggerated with the development of medical procedures such as anesthesia and caesarean section [22]. Presently, the increased use of ultrasounds, fetal heart monitors, and increasing caesarean delivery rates illustrate multiple ways that women's pregnancy and childbirth experiences have been heavily medicalized [22-24].

\subsubsection{Medicalization of Childbirth}

Medicalization is defined as the tendency to pathologize normal bodily processes and states, resulting in unnecessary medical management [25]. Some factors influencing childbirth medicalization include the complexity of maternal health care, culture of medical dominance, submissive culture in nursing and midwifery [26], technological advancement (e.g. emergence of biomedicine) [27, 28], and focus on risks involved with natural childbirth [29]. Childbirth medicalization is apparent in the widespread and increasing rates of medical interventions (i.e. caesarean and instrumental deliveries). In Western coun- 
tries (e.g. the United States, Italy, and the United Kingdom), caesarean births account for approximately $20 \%$ of childbirth procedures $[22,30]$. In Spain, the extent of medicalization is demonstrated in some of the highest caesarean delivery rates in Europe (e.g. $40 \%$ increase of the procedure in Catalonia over 5 years) and obstetricians are held accountable for not allowing women to be involved in childbirth decision-making [22, 31]. Furthermore, Eastern countries such as China and Singapore have one of the highest caesarean delivery rates in the world and South East Asia, respectively [32, 33]. In China, caesarean rates in the 90 s rose from below $5 \%$ to above $10 \%$, with urban rates as high as $20 \%$ by 1996 . In 2010, of the 16 million babies born in China, approximately $50 \%$ were born by caesarean delivery [32]. In Singapore, the overall caesarean delivery rate increased from $32.2 \%$ (Year 2005) to $37.4 \%$ in year 2014 [34]. Some contributing factors to the alarming rates of caesarean delivery in China and Singapore include the obstetric care system (i.e. urbanization, medicalization of childbirth, and financial incentives), provider factors (i.e. obstetric training and staffing), and patient factors (i.e. parity where primiparas prefer caesarean delivery as it is deemed as more effective, and education level where universityeducated women prefer caesarean births) [32].

The potential impacts of medicalization of childbirth and pregnancy include (1) dependence on medicine and the medical field [35], (2) hampering the embodiment process that neglects the body as somato-psychic [36, 37], and (3) hindering the gift dynamic at play during natural childbirth [38]. The medicalization of childbirth, at times can be a life-saving and effective procedure, but if done unnecessarily it may put women and their babies at increased susceptibility to mortality and morbidity and has aversive effects on maternal health and pregnancy outcomes [39, 40]. Hence, it should be limited to instances of medical emergencies. Finally, the medicalization of childbirth and pregnancy is coupled with a negative connotation, in which natural pregnancy and childbirth are now conceptualized as illnesses or diseases that require medical technologies and interventions [23, 41].

\subsubsection{Latest Move Toward Natural Physiological Birth}

In response to the increasing rates of medical intervention and medicalization of childbirth and pregnancy, there has been increasing efforts in promoting natural childbirth [42]. The World Health Organization (1997) highlighted the need to eliminate unnecessary medical interventions in childbirth [43] and countries have passed policies, initiatives, and guidelines to promote and protect natural childbirth. In Australia, the current maternity care reform (National Maternity Services Plan) is grounded on the underlying philosophy of childbirth as a natural physiological process [44]. Guidelines to protect, promote, and support natural childbirth were established and published in Queensland [45]. A policy in New South Wales (Towards Normal Birth in New South Wales) required all birthing facilities and institutions in the Australian state to have a written policy for natural childbirth by 2015 [46]. In the United Kingdom (UK), the Royal College of Midwives established the Campaign for Normal Birth that is now integrated as part of the Better Births Initiative [47]. Health care providers in Canada published a Joint Policy Statement on Normal Birth to protect the practice of natural birth [48]. In the Netherlands, the maternity care system is grounded on the principle that childbirth and pregnancy are natural physiological processes and community-based midwifery continue to play an important role [49] facilitating natural birth and continuity of care after childbirth. Hence, home birth remains a widely accepted and well-integrated part of the health care system [50].

Researchers have explored the role of midwives in encouraging natural birth as midwiferyled models of care are associated with the reduced use of medical interventions and mothers' increased satisfaction with the natural birthing experience [51]. Thompson et al.'s (2016) qualitative study explored Dutch midwives' attitudes and motivations toward the promotion of natural childbirth, and identified factors associated with these attitudes and motivations [52]. Findings revealed that midwives perceive the safeguarding 
and promotion of natural physiological childbirth as the focus of their role, and hospital culture is deemed as a barrier to practices that promote natural childbirth [52]. To overcome this barrier, midwives expressed the need to be aware of factors that inhibit and encourage natural childbirth practices, and to employ strategies that promote natural childbirth in home and hospital settings [52]. A recent UK evaluation study examined the effectiveness of an educational training package designed for midwives and maternity support workers [53]. The training package included a core workshop entitled "Keeping Birth Normal" (KBM), workshops that focused on antenatal education, communication skills, and baby massage [53]. Findings revealed that midwives were appreciative of the educational materials (e.g. videos and lectures) and expressed that small group discussions helped to facilitate learning [53]. They described two barriers to the implementation of training: (1) cultural focus on risk and (2) hospital culture of low prioritization of natural childbirth $[29,52,53]$. Despite the barriers, the training provided opportunities (1) to build a community of practice around natural childbirth that helped in overcoming the existing risk-focused culture and (2) created awareness within the midwifery unit that the promotion of natural physiological childbirth is central to their role [53]. These evidences urge the need for future midwifery education and research to focus on developing and testing strategies that support midwives in delivery health-promoting care and services [52, 53].

Moreover, a recent qualitative study that aimed to clarify how primiparous women in Turkey experience childbirth and intrapartum care revealed that the women wanted a natural birth without interventions [54]. Grounded theory guided interviews with 12 women were conducted and they reported wanting vaginal birth without interventions, which required empowerment and social support from others (i.e. health care professionals, family, and friends) [54]. Also, they expressed that routine medical interventions during the birth process cause their anxiety and frustration [54]. These interventions become obstructions to women's natural posi- tion during birth, interrupt the birth process, and compromise their dignity and sense of autonomy [54]. This is an exceptionally important finding as Turkey has one of the highest caesarean rates, in which care during labor often entails the overuse of medicalized interventions such as episiotomy (i.e. experienced by $93.3 \%$ of primiparous women in Turkey) and augmentation with oxytocin and caesarean section [55]. With women who voiced their "want" for natural birth without interventions due to the negative consequences of medicalized interventions, it is a "need" to work toward a less- or non-medicalized childbirth process through positive health promotion.

With the progression toward less-medicalized models of birth through promotion of natural childbirth, existing barriers such as hospital and risk-focused culture are still apparent in many parts of the health care system. Therefore, it is imperative to adopt an appropriate approach of health promotion that will provide the necessary shift of focus from the risks and complications associated with childbirth to one that provides a positive and health-promoting experience for families with a newborn [56].

\subsection{Health Promotion: Use of Salutogenesis Theory}

Health promotion is purported to enable individuals with increased control over their own health [57]. One such approach is to integrate the Salutogenesis theory that was coined by the medical sociologist Antonovsky (1979) who focused on the origin of good health rather than the origin of illness [58]. Salutogenesis illustrates that the state of health lies on a spectrum-from complete absence of health to absolute state of health on the other extreme [58]. This theory implies that one has the ability and control to move toward better health with available resources around them. Salutogenesis consists of two: (1) generalized resistance resources (GRR), i.e. characteristics of an individual, family, or community that are resources to aid the individual in coping with stressors and (2) sense of coherence $(S O C)$, i.e. one's ability to use available resources 


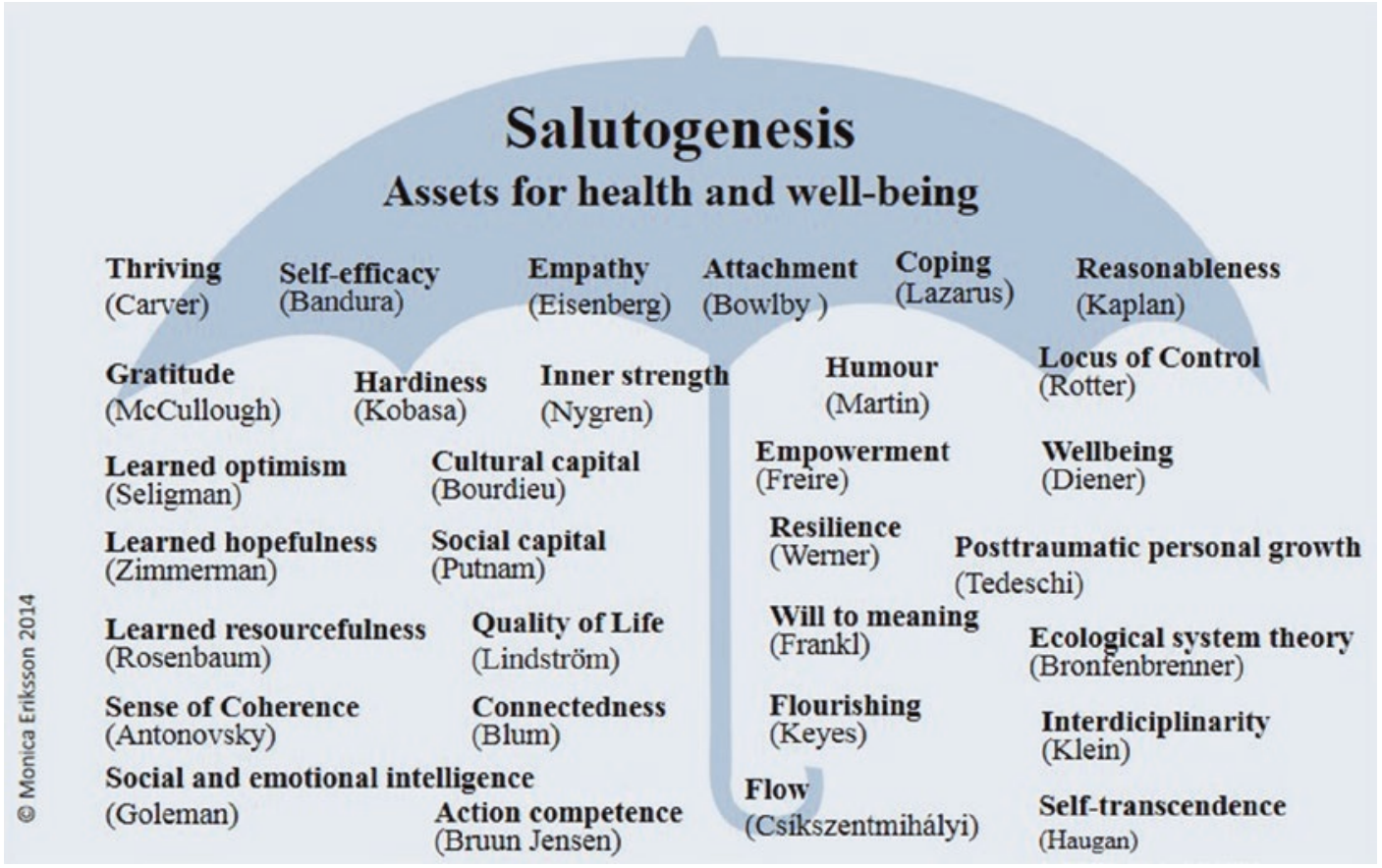

Fig. 14.1 Salutogenesis Umbrella (Reproduced with permission from Folkhälsan Research Center, Lindstrom \& Eriksson, 2010) [65] According to Monica Eriksson, there is a revised version of the Salutogenesis umbrella which is used now)

ing one's ability to mobilize both internal and external resources for well-being [66, 67]. More recently, Johansson et al. (2018) developed and implemented a salutogenic treatment model in a clinical setting of emergency child and adolescent psychiatry in Sweden [68]. Eight GRRs (e.g. clear language, daily information, and participation in decision-making) were developed and implemented in the emergency unit, and parents reported increased satisfaction with the treatment and care provided [68]. Mental health of children improved during their stay at the hospital, and results revealed reduced treatment length and readmission rates [68]. Therefore, the salutogenic framework can be applied as a strong and effective theoretical basis to direct the development and implementation of health care services.

Multiple studies have adopted the salutogenic framework to highlight important qualities of health care providers and areas of improvements to promote SOC in new mothers [69-71]. Findings revealed that midwives should stay calm in tight time-constraint environments which it can be an asset that operates by increas- 
while targeting positive wellness topics rather than risk factors and provide mothers constant reassurances to encourage SOC [69]. Dahlberg et al. (2016) interviewed new mothers and they expressed the need to be cared for exclusively and highlighted the importance of a midwife for emotional support, coaching, and parenting guidance [70]. In Kelly et al. (2016) study, Salutogenesis was adopted, and a framework was proposed to promote families' SOC and well-being to better facilitate their transition into parenthood [71]. To promote SOC, the health care sector should (1) improve on the continuity of care and support for parents in the postpartum period, (2) improve the method of information delivery, and (3) increase parental involvement in decision-making, while balancing relationships among parents and health care professionals [71]. Altogether, these salutogenically focused studies highlighted the need for health care providers to possess more humane qualities (i.e. midwives to stay calm and provide emotional support), as opposed to just the delivery of tangible materials (e.g. provision of educational booklets) to provide positive and health-promoting services.

\subsubsection{Salutogenesis Theory in Perinatal Health Care}

The salutogenic framework has been applied in perinatal health care, specifically for families having a newborn. Existing salutogenically focused studies addressed several phenomena across the perinatal period. Greer et al. (2014) explored mothers' fear of childbirth and its impact on birth choices among women and their partners through interviews carried out within the SOC theoretical framework [72]. The findings (i.e. riskiness, ways of coping, and being a good parent) were related to the three dimensions of SOC (i.e. comprehensibility, manageability, and meaningfulness) [72]. For instance, pregnant couples feared the repercussions of natural childbirth and preferred medical interventions over natural childbirth to cope with uncertainties and to ensure a smooth and safe transition to parenthood [72]. The three dimensions of SOC were utilized to demonstrate parental and professional perspectives on neonatal care [73]. The concepts of comprehensibility, manageability, and meaningfulness provided a theoretical foundation to develop and integrate meaningful care that complemented existing services for optimal care [73]. Similarly, Kelly et al. (2016) applied the salutogenic framework to discuss ways in maintaining parental mental well-being during the perinatal period [71]. It was reported that the framework shaped parental mental well-being in two ways: (1) through helping parents to make sense of parenting stressors by examining psychosocial protective factors such as optimism, self-esteem, and stressor identification as well as how these factors fit into their lives, and they can use them to cope with the parenting stressors, and (2) strengthening parents' sense of coherence [71]. As such the salutogenic framework could be used as a perinatal parent education framework to promote overall parental wellbeing during the perinatal period [71]. There are only two existing reviews that consolidated existing studies that examined Salutogenesis in perinatal health care. Smith et al. (2014) identified salutogenically focused outcomes $(n=16$; e.g. maternal satisfaction and breastfeeding) and non-salutogenically focused outcomes ( $n=49$; e.g. measures of neonatal morbidity) during the intrapartum period (Smith et al. 2014). The review implied a lack of salutogenically focused outcomes reported in intrapartum interventionbased research [74]. Perez-Botella et al. (2015) examined how the Salutogenesis theory can be utilized to address several parenting outcomes across the perinatal period, and implied that the theory is rarely used in maternity care research and urged future research to measure salutogenitcally oriented outcomes to provide a balance in maternity care design [75]. Though there are increasing but limited perinatal studies that adopted the salutogenic framework with majority of studies focussing on only SOC, there is a crucial need to expand the utilization of the salutogenic framework and concepts in the salutogenic umbrella (other than SOC) to ensure a holistic application of the theory to encourage health promotion during the perinatal period. 


\subsubsection{Managing the Perinatal Period with Physical Activity: A Salutogenic Approach}

Women during pregnancy are generally sedentary and could gain excessive weight due to physical limitations, increased appetite, and tiredness [76, 77]. As a result, majority of the pregnant women fail to meet the recommended level of physical activity and this increases their risk of negative pregnancy conditions such as gestational diabetes and pregnancy-induced hypertension $[77,78]$. Also, the children of obese women during pregnancy are more likely to become obese adults [79]. Therefore, there is a need for health care professionals to inform pregnant couples about healthy lifestyle choices such as the engagement in physical activity for health promotion during the perinatal period [78]. Physical activity is recommended as a health promoting approach that aid pregnant couples to cope with the bodily changes throughout the perinatal period [80]. Also, physical activity has been found to be associated with increased overall well-being during pregnancy, self-esteem, shorter duration of labor, fewer depressive symptoms, higher prevalence of natural childbirth [77], and reduced occurrence of gestational diabetes [81] and pregnancy-induced hypertension [82].

The Salutogenesis theory has been found useful in directing families to engage in physical activity as a mean to cope with potential negative pregnancy conditions during the perinatal period [83]. Frequent physical activity has been shown to be associated with strong SOC [83]. In Hassmen et al.'s (2000) population study significant correlations were found between individuals, both men and women, who exercised more frequently with higher SOC than those who exercise less frequently [84]. Another study from the Netherlands revealed that individuals with a strong SOC engaged in sports more frequently and have better health outcomes such as lower average blood pressure [85]. The individuals with increased physical activity not only found to have stronger SOC but also higher self-esteem, stronger control over bad habits, and more positive attitudes toward changes in life [86]. A review conducted by Eriksson and Lindstrøm (2006) synthesized empirical findings on SOC and reported that stronger SOC is linked to better health outcomes [87]. Stronger SOC was found to be associated with lower mortality risk [85], delay onset of cancer [88], and lower rates of diabetes [89].

During the perinatal period, one of the main contributing factor that lead to physical inactivity is lack of social support [76, 78]. Women reported that due to lack of support from their partners to run errands at home and to care for older children, they often have limited time and tend to neglect their physical exercise. To promote a strong social support and to increase physical activity, the salutogenic framework can pave the path in encouraging families to engage in physical activities together. On top of the two main components (i.e. GRR and SOC) of the salutogenic theory, the other concepts from the salutogenic umbrella such as connectedness [61], coping [63], and well-being [62], can be included to form a conceptual framework in the development of family-focused interventions catered for the pregnant couples. For instance, during prenatal care, health care professionals (i.e. doctors and midwives) have the unique opportunity to inform pregnant couples about the benefits of healthy lifestyle choices such as walking and swimming and to recommend individualized exercise routines and nutritional plans for couples to follow through. At this stage, pregnant couples can be motivated to have a shared physical activity plan to enhance their connectedness [61] and thus supporting each other for a shared activity that will eventually benefit them both physically and mentally. These individualized couple-based exercise routines and nutritional plans can be then enhanced with other shared physical and mental changes with the addition of the newborn during the postpartum period.

During postpartum, shared physical activities and nutritional plans could continue to direct couples and their newborn through connecting and benefitting the overall familial well-being. Hence, health care professionals who recommend physical activities and nutritional plans (Coping) help to provide a common ground for 
both mothers and fathers (GRR) with a newborn (Connectedness) to manage the likelihood of negative pregnancy conditions. The adherence to exercise routines and nutritional plans could aid in parental overall physical and mental wellbeing (Well-being; SOC), improving their physical and mental health. With connectedness and appropriate coping to stressors throughout the perinatal period, the overall familial well-being and SOC could be maintained using the available resources, and thus, result in positive perinatal health outlooks and outcomes. Therefore, additional concepts in the salutogenic umbrella can be included on top of GRR and SOC in directing the development of family-focused services.

\subsection{Literature Gaps, Implications, and Future Research}

With rising prevalence rates of negative parental mental health during the perinatal period, mothers and fathers reported negative feelings and expressed needs to assist them during this period. The medicalization of childbirth resulted in aversive effects on maternal health and pregnancy outcomes and is coupled with a negative connotation that pregnancy and childbirth are conceptualized as illnesses or diseases. Despite the increasing efforts to promote natural physiological childbirth, hospital and risk-focused cultures continue to pose as strong barriers to normalizing natural childbirth. Therefore, these pose as existing gaps in the literature that imply a necessary shift of focus to one that provides a positive and health-promoting experience for families with a newborn. To bridge these gaps, health promotion that integrates the Salutogenesis theory forms a foundational basis that directs health care professionals and providers to design and deliver wellbeing focused interventions and care services for the pregnant couples and the new parents. Existing salutogenically focused studies that adopted quantitative (i.e. correlational studies and randomized controlled trials) and/or qualitative approaches (i.e. in-depth interviews and grounded-theory studies), implied that the saluto- genic framework is a potential school of thought that urges health care professionals to adopt in providing positive and health-promoting services and care to assist families during the perinatal period. Also, salutogenically focused qualitative studies that interviewed pregnant women and midwives during the perinatal period highlighted the need for health care providers to possess more humane qualities (e.g. emotional support) to deliver positive and health-promoting care. This implies the need to train health care professionals such as midwives to be prepared and proactive in delivering optimal positive perinatal care. Future research can consider the salutogenic framework in designing midwifery education that focuses on developing and testing strategies to support midwives in this endeavour. The salutogenic framework is well-positioned as a perinatal parent education framework and future research can aim to design salutogenically focused interventions (e.g. support groups and perinatal classes) to promote parental well-being during the perinatal period.

Existing quantitative and correlational studies have shown the benefits of being physically active during the perinatal period, but the majority remains physically inactive. This implies the need for health care professionals to inform pregnant couples about healthy lifestyle choices for health promotion and prevention of negative pregnancy conditions during the perinatal period. Future research can aim to adopt the salutogenic framework in developing family-focused care services (i.e. exercise routines and nutritional plans) catered to pregnant couples during the perinatal period. It is also important to conduct further research on pregnant couples and factors that promote physical activity.

\subsection{Conclusion}

There are increasing but limited perinatal studies that adopted the salutogenic framework, especially elements of SOC in isolation. Future research could expand the utilization of the framework and concepts in the salutogenic umbrella beyond SOC to ensure a holistic application of 
the theory to encourage health promoting outcomes during the perinatal period. As majority of the existing studies are from the West, more studies from geographically diverse backgrounds such as the Middle East and Asia are required to explore and garner a holistic view of salutogenic framework and health promotion across the perinatal period. In summary, salutogenic theory offers the potential to influence a shift away from negative health outlooks and outcomes, medicalization of childbirth, toward health promotion and positive well-being focus for maternity care services design and delivery in the future.

\section{Take-Home Messages}

- It is imperative to enhance quality of care for families having a newborn to ensure optimal parental mental health and newborn health.

- A fundamental step is to recognize and acknowledge the problem of medicalization interventions during the perinatal period, and to adopt an appropriate approach of health promotion that will provide a shift of focus from the risks and complications associated with childbirth to one that is positive and health-promoting for families with a newborn.

- It is timely to evaluate and implement the upand-coming salutogenic framework in health care services that focuses on positive wellbeing and health promotion, especially with its increasing but limited application in perinatal health care. The framework serves as a guide for health care professionals in developing health-promoting services to families with a newborn.

- There is a crucial need to expand and integrate the salutogenic framework in promoting the engagement in physical activities as families tend to become sedentary during the perinatal period. The framework serves as a common ground for health care professionals and families to discuss individualized activity plans that can optimize their experience and positive well-being during the perinatal period.

- Theory-based research is the way to describe and evaluate evidence-based quality care to families with a newborn. Health care professionals need to be equipped with relevant skills in conducting quality research that work toward the expansion of the framework and concepts in the salutogenic umbrella beyond SOC to ensure a holistic application of the theory to encourage health promoting outcomes during the perinatal period.

\section{References}

1. Leach LS, Poyser C, Cooklin AR, Giallo R. Prevalence and course of anxiety disorders (and symptom levels) in men across the perinatal period: a systematic review. J Affect Disord. 2016;190:675-86.

2. Duncan LG, Bardacke N. Mindfulness-based childbirth and parenting education: promoting family mindfulness during the perinatal period. J Child Fam Stud. 2010;19(2):190-202.

3. Buultjens M, Murphy G, Robinson P, Milgrom J. The perinatal period: a literature review from the biopsychosocial perspective. Clin Nurs Stud. 2013;1(3):19-31.

4. Gavin NI, Gaynes BN, Lohr KN, Meltzer-Brody S, Gartlehner G, Swinson T. Perinatal depression: a systematic review of prevalence and incidence. Obstet Gynecol. 2005;106(5 Pt 1):1071-83.

5. Leach LS, Poyser C, Fairweather-Schmidt K. Maternal perinatal anxiety: a review of prevalence and correlates. Clin Psychol. 2017;21(1):4-19.

6. Gao L, Chan SW, Mao Q. Depression, perceived stress, and social support among first-time Chinese mothers and fathers in the postpartum period. Res Nurs Health. 2009;32(1):50-8.

7. Paulson JF, Bazemore SD. Prenatal and postpartum depression in fathers and its association with maternal depression: a meta-analysis. JAMA. 2010;303(19): 1961-9.

8. Cameron EE, Sedov ID, Tomfohr-Madsen LM. Prevalence of paternal depression in pregnancy and the postpartum: an updated meta-analysis. J Affect Disord. 2016;206:189-203.

9. Philpott LF, Savage E, FitzGerald S, Leahy-Warren P. Anxiety in fathers in the perinatal period: a systematic review. Midwifery. 2019;76:54-101.

10. Nagle U, Farrelly M. Women's views and experiences of having their mental health needs considered in the perinatal period. Midwifery. 2018;66:79-87.

11. Ong SF, Chan W-CS, Shorey S, Chong YS, KlaininYobas P, He H-G. Postnatal experiences and support needs of first-time mothers in Singapore: a descriptive qualitative study. Midwifery. 2014;30(6): 772-8.

12. Edwards E, Timmons S. A qualitative study of stigma among women suffering postnatal illness. J Ment Health. 2005;14(5):471-81.

13. Slade P, Morrell CJ, Rigby A, Ricci K, Spittlehouse J, Brugha TS. Postnatal women's experiences of man- 
agement of depressive symptoms: a qualitative study. Br J Gen Pract. 2010;60(580):e440-e8.

14. Darwin Z, Galdas P, Hinchliff S, Littlewood E, McMillan D, McGowan L, et al. Fathers' views and experiences of their own mental health during pregnancy and the first postnatal year: a qualitative interview study of men participating in the UK Born and Bred in Yorkshire (BaBY) cohort. BMC Pregnancy Childbirth. 2017;17(1):45.

15. Finnbogadóttir H, Crang Svalenius E, Persson E. Expectant first-time fathers' experiences of pregnancy. Midwifery. 2003;19(2):96-105.

16. Iwata H. Experiences of Japanese men during the transition to fatherhood. J Transcult Nurs. 2014;25(2):159-66.

17. Poh HL, Koh SSL, Seow HCL, He H-G. First-time fathers' experiences and needs during pregnancy and childbirth: a descriptive qualitative study. Midwifery. 2014;30(6):779-87.

18. Rowe HJ, Holton S, Fisher JRW. Postpartum emotional support: a qualitative study of women's and men's anticipated needs and preferred sources. Aust J Prim Health. 2013;19(1):46.

19. Edhborg M, Carlberg M, Simon F, Lindberg L. Waiting for better times: experiences in the first postpartum year by Swedish fathers with depressive symptoms. Am J Mens Health. 2016;10(5): 428-39.

20. Pålsson P, Persson EK, Ekelin M, Kristensson Hallström I, Kvist LJ. First-time fathers experiences of their prenatal preparation in relation to challenges met in the early parenthood period: implications for early parenthood preparation. Midwifery. 2017;50:86-92.

21. Shorey S, Dennis CL, Bridge S, Chong YS, Holroyd E, He HG. First-time fathers' postnatal experiences and support needs: a descriptive qualitative study. J Adv Nurs. 2017;73(12):2987-96.

22. Johanson R, Newburn M, Macfarlane A. Has the medicalisation of childbirth gone too far? BMJ. 2002;324(7342):892-5.

23. Parry DC. "We wanted a birth experience, not a medical experience": exploring Canadian Women's use of midwifery. Health Care Women Int. 2008;29(8-9):784-806.

24. Mitchell LM. Baby's first picture: ultrasound and the politics of fetal subjects. Toronto: University of Toronto Press; 2001.

25. Inhorn MC. Defining Women's Health: a dozen messages from more than 150 ethnographies. Med Anthropol Q. 2006;20(3):345-78.

26. Clesse C, Lighezzolo-Alnot J, de Lavergne S, Hamlin S, Scheffler M. The evolution of birth medicalisation: a systematic review. Midwifery. 2018;66:161-7.

27. Nye RA. The evolution of the concept of medicalization in the late twentieth century. J Hist Behav Sci. 2003;39(2):115-29.

28. Saintôt B. More managing for pregnancy and childbirth? [In French: Gérer toujours plus la grossesseet 1'accouchement ?]. Laennec. 2015;63(4):6-15.
29. Parry DC. Women's lived experiences with pregnancy and midwifery in a Medicalized and Fetocentric context: six short stories. Qual Inq. 2006;12(3):459-71.

30. Thomas J, Paranjothy S. The national sentinel caesarean section audit report. National Sentinel Caesarean Section Audit Report. 2001.

31. Topçu S, Brown P. The impact of technology on pregnancy and childbirth: creating and managing obstetrical risk in different cultural and socio-economic contexts. Health Risk Soc. 2019;21(3-4):89-99.

32. Hellerstein S, Feldman S, Duan T. China's 50\% caesarean delivery rate: is it too high? BJOG. 2015;122(2):160-4.

33. Hou L, Hellerstein S, Vitonis A, Zou L, Ruan Y, Wang $\mathrm{X}$, et al. Cross sectional study of mode of delivery and maternal and perinatal outcomes in mainland China. PLoS One. 2017;12(2):e0171779.

34. Chi C, Pang D, Aris IM, Teo WT, Li SW, Biswas A, et al. Trends and predictors of cesarean birth in Singapore, 2005-2014: a population-based cohort study. Birth. 2018;45(4):399-408.

35. Chanial P. The fugitive moment in which society takes: the gift, the game and the whole. [in French: L'instant fugitif où la société prend. Le don, la partie et le tout.]. Revue du MAUSS semestrielle. 2010;36:521-38.

36. Davis-Floyd R. The technocratic, humanistic, and holistic paradigms of childbirth. Int J Gynecol Obstet. 2001;75:S5-S23.

37. Shabot SC. Making loud bodies "feminine": a feminist-phenomenological analysis of obstetric violence. Hum Stud. 2016;39(2):231-47.

38. Azcue M, Tardif J. Begetting in the perspective of gift: what it means to give birth in our modern health care system. Revue du MAUSS. 2012;39(1):163-79.

39. Rosen T. Placenta accreta and cesarean scar pregnancy: overlooked costs of the rising cesarean section rate. Clin Perinatol. 2008;35(3):519-29. x

40. Silver RM, Landon MB, Rouse DJ, Leveno KJ, Spong CY, Thom EA, et al. Maternal morbidity associated with multiple repeat cesarean deliveries. Obstet Gynecol. 2006;107(6):1226-32.

41. Walters V. Women's perceptions regarding health and illness. In: Bolaria BS, Dickinson HD, editors. Health, illness and health care in Canada. Toronto: Hartcourt Brace; 1994. p. 307-25.

42. Prosser SJ, Barnett AG, Miller YD. Factors promoting or inhibiting normal birth. BMC Pregnancy Childbirth. 2018;18(1):241-10.

43. Technical Working Group WHO. Care in Normal Birth: a practical guide. Birth. 1997;24(2):121-3.

44. Conference AHM. National maternity services plan 2010. 2010.

45. Program QMaNCG. Normal birth. 2012.

46. Health NSW. Towards normal birth in NSW. 2010.

47. Midwives TRCo. Better birth initiative. 2018.

48. Joint Policy Statement on Normal Childbirth. J Obstet Gynaecol. 2008;30(12):1163-5.

49. De Vries R, Press AU. Pleasing birth: midwives and maternity care in the Netherlands. Amsterdam: Amsterdam University Press; 2005. 
50. Christiaens W, Nieuwenhuijze MJ, de Vries R. Trends in the medicalisation of childbirth in Flanders and the Netherlands. Midwifery. 2013;29(1):e1-8.

51. Sandall J, Soltani H, Gates S, Shennan A, Devane D, Sandall J. Midwife-led continuity models versus other models of care for childbearing women. Cochrane Database Syst Rev. 2016;2016(4):CD004667.

52. Thompson SM, Nieuwenhuijze MJ, Low LK, de Vries R. Exploring Dutch midwives' attitudes to promoting physiological childbirth: a qualitative study. Midwifery. 2016;42:67-73.

53. Walker S, Batinelli L, Rocca-Ihenacho L, McCourt C. 'Keeping birth normal': exploratory evaluation of a training package for midwives in an inner-city, alongside midwifery unit. Midwifery. 2018;60:1-8.

54. Deliktas Demirci A, Kabukcuglu K, Haugan G, Aune I. "I want a birth without interventions": women's childbirth experiences from Turkey. Women Birth. 2019;32(6):e515.

55. Kartal B, Kizılırmak A, Calpbinici P, Demir G. Retrospective analysis of episiotomy prevalence. J Turk German Gynecol Assoc. 2017;18(4):190-4.

56. Sinclair M, Stockdale J. Achieving optimal birth using salutogenesis in routine antenatal education. Evid Based Midwif. 2011;9(3):75.

57. WHO. What is health promotion. 2016.

58. Antonovsky A. Health, stress, and coping. London: Jossey-Bass; 1979.

59. Antonovsky A. The salutogenic model as a theory to guide health promotion. Health Promot Int. 1996;11(1):11-8.

60. Bandura A. Self-efficacy: the exercise of control. New York: W.H. Freeman; 1997.

61. Blum RJMCI. School connectedness: improving students' lives; 2005. p. 1-18.

62. Diener E. The science of well-being: the collected works of Ed Diener. 1. Aufl.;1; ed. Dordrecht: Springer; 2009.

63. Folkman S, Lazarus RS. Coping as a mediator of emotion. J Pers Soc Psychol. 1988;54(3):466-75.

64. McCullough ME, Emmons RA, Tsang J-A. The grateful disposition: a conceptual and empirical topography. J Pers Soc Psychol. 2002;82(1):112-27.

65. Lindström B, Eriksson M. The hitchhiker's guide to salutogenesis: Salutogenic pathways to health promotion. Folkhälsan Research Center, Health Promotion Research; 2010.

66. Garcia-Moya I, Morgan A. The utility of salutogenesis for guiding health promotion: the case for young people's well-being. Health Promot Int. 2017;32(4):723-33.

67. Morgan A, Hernán M. Promoting health and wellbeing through the asset model. Revista Española de Sanidad Penitenciaria. 2013;15(3):78-86.

68. Johansson BA, Pettersson K, Tydesten K, Lindgren A, Andersson C. Utvecklingsrelaterade störningar asoåib-ou, et al. implementing a salutogenic treatment model in a clinical setting of emergency child and adolescent psychiatry in Sweden. J Child Adolesc Psychiatr Nurs. 2018;31(2-3):79-86.
69. Browne J, O’Brien M, Taylor J, Bowman R, Davis D. 'You've got it within you': the political act of keeping a wellness focus in the antenatal time. Midwifery. 2014;30(4):420-6.

70. Dahlberg U, Persen J, Skogas AK, Selboe ST, Torvik HM, Aune I. How can midwives promote a normal birth and a positive birth experience? The experience of first-time Norwegian mothers. Sex Reprod Healthc. 2016;7:2-7.

71. Kelly RG, Hauck Y, Thomas S. Salutogenesis: a framework for perinatal mental wellbeing. Aust $\mathrm{J}$ Child Fam Health Nurs. 2016;13(2):4.

72. Greer J, Lazenbatt A, Dunne L. 'Fear of childbirth' and ways of coping for pregnant women and their partners during the birthing process: a salutogenic analysis. Evid Based Midwif. 2014;12:95-100.

73. Thomson G, Moran VH, Axelin A, Dykes F, Flacking $\mathrm{R}$, Högskolan D, et al. Integrating a sense of coherence into the neonatal environment. BMC Pediatr. 2013;13(1):84.

74. Smith V, Daly D, Lundgren I, Eri T, Benstoem C, Devane D, et al. Salutogenically focused outcomes in systematic reviews of intrapartum interventions: a systematic review of systematic reviews. Midwifery. 2014;30(4):e151-e6.

75. Perez-Botella M, Downe S, Meier Magistretti C, Lindstrom B, Berg M, Sahlgrenska A, et al. The use of salutogenesis theory in empirical studies of maternity care for healthy mothers and babies. Sex Reprod Healthc. 2014;6(1):33-9.

76. Gaston A, Cramp A. Exercise during pregnancy: a review of patterns and determinants. J Sci Med Sport. 2011;14(4):299-305.

77. Gjestland K, Bø K, Owe K, Eberhard-Gran M. Do pregnant women follow exercise guidelines? Prevalence data among 3482 women, and prediction of low-back pain, pelvic girdle pain and depression. Br J Sports Med. 2012;47:515-20.

78. Birkheim SL. Association between sense of coherence and participation in a free offer of exercise for pregnant women. 2015.

79. de Jersey SJ, Nicholson JM, Callaway LK, Daniels LA. An observational study of nutrition and physical activity behaviours, knowledge, and advice in pregnancy. BMC Pregnancy Childbirth. 2013;13:115.

80. Cioffi J, Schmied V, Dahlen H, Mills A, Thornton C, Duff $\mathrm{M}$, et al. Physical activity in pregnancy: women's perceptions, practices, and influencing factors. J Midwifery Womens Health. 2010;55(5):455-61.

81. Dempsey JC, Sorensen TK, Williams MA, Lee IM, Miller RS, Dashow EE, et al. Prospective study of gestational diabetes mellitus risk in relation to maternal recreational physical activity before and during pregnancy. Am J Epidemiol. 2004;159(7):663-70.

82. Sorensen TK, Williams MA, Lee IM, Dashow EE, Thompson ML, Luthy DA. Recreational physical activity during pregnancy and risk of preeclampsia. Hypertension. 2003;41(6):1273-80.

83. Kuuppelomäki M, Utriainen P. A 3 year follow-up study of health care students' sense of coherence and 
related smoking, drinking and physical exercise factors. Int J Nurs Stud. 2003;40(4):383-8.

84. Hassmen P, Koivula N, Uutela A. Physical exercise and psychological well-being: a population study in Finland. Prev Med. 2000;30(1):17-25.

85. Super S, Verschuren WMM, Zantinge EM, Wagemakers MAE, Picavet HSJ. A weak sense of coherence is associated with a higher mortality risk. J Epidemiol Community Health. 2014;68(5):411-7.

86. Pallant JF, Lae L. Sense of coherence, well-being, coping and personality factors: further evaluation of the sense of coherence scale. Personal Individ Differ. 2002;33(1):39-48.
87. Eriksson M, Lindström B, Högskolan V, Institutionen för omvårdnad hok, Avd för hälsa och k. Validity of Antonovsky's sense of coherence scale: a systematic review. J Epidemiol Community Health. 2005;59(6):460-6.

88. Poppius E, Virkkunen H, Hakama M, Tenkanen L. The sense of coherence and incidence of cancer-role of follow-up time and age at baseline. J Psychosom Res. 2006;61(2):205-11.

89. Kouvonen AM, Väänänen A, Woods SA, Heponiemi T, Koskinen A, Toppinen-Tanner S. Sense of coherence and diabetes: a prospective occupational cohort study. BMC Public Health. 2008;8(1):46.

Open Access This chapter is licensed under the terms of the Creative Commons Attribution 4.0 International License (http://creativecommons.org/licenses/by/4.0/), which permits use, sharing, adaptation, distribution and reproduction in any medium or format, as long as you give appropriate credit to the original author(s) and the source, provide a link to the Creative Commons license and indicate if changes were made.

The images or other third party material in this chapter are included in the chapter's Creative Commons license, unless indicated otherwise in a credit line to the material. If material is not included in the chapter's Creative Commons license and your intended use is not permitted by statutory regulation or exceeds the permitted use, you will need to obtain permission directly from the copyright holder. 